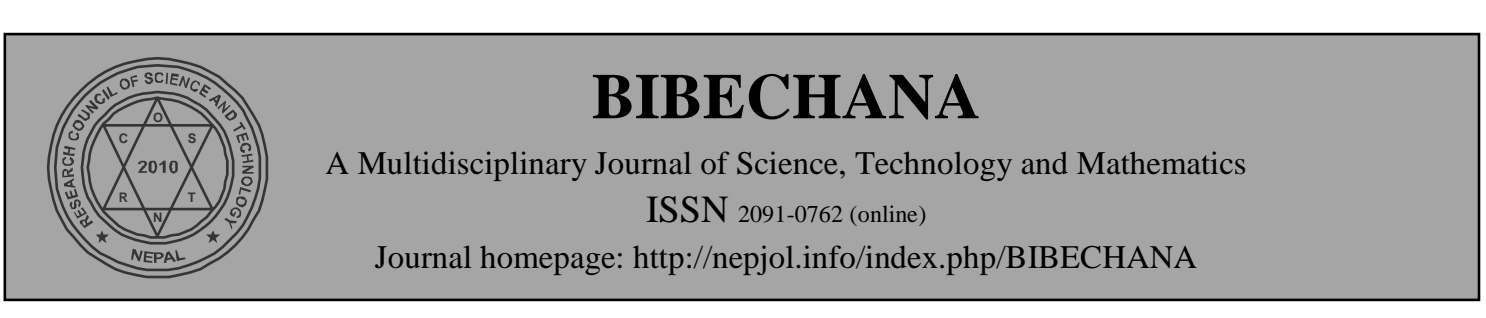

\title{
Some remarks on the definition of numbers
}

\author{
G. K. Palei ${ }^{1}$, P. K. Karn ${ }^{2 *}$ \\ ${ }^{1}$ Dept. of Mathematics, B.N. College Patna, Patna University, Patna, India \\ ${ }^{2}$ Dept. of Mathematics, M.M.A.M. Campus, Biratnagar, T.U., Nepal \\ *Corresponding Author Email: pawankarn12345@gmail.com
}

Accepted for publication: February 15, 2014

\begin{abstract}
In this paper some remarks have been given on the definition of number. It is clear that Peano's definition of a number and Frege-Russel definition of number are not satisfactory. Number states some things about the concept and not about the counted things themselves.
\end{abstract}

() 2014 RCOST: All rights reserved.

Keywords: Quotient Number; Polygamy; Successor.

\section{Vaisesika definition of number}

The Vaisesikas have attempted to define and characterized numbers unity, duality, triplicity etc and that they have accepted numbers as objectively real quantities inhering in all substances. For them unity, like God, inheres in all substances and is a basic number with which all higher numbers are formed by means of relative understanding Modern Western Philosopher like Hilbert and Brouwer also agree with their view that the concept of one is fundamental in mathematics. But the Vaisesikas have not defined zero, which has its use as a number in the Indian Mathematical literatures since about 200 B.C. [1] or even earlier in the Vedic literature. It was Brahmagupta (628 A.D.), the prince of Indian mathematics, who correctly defined zero. He gave a relational rather than half-scientific and half- intuitive definition of zero. Since zero is the number smaller than any one of the numbers unity, duality etc. which are used for counting purposes, it is desirable and proper to define zero first and then to define other numbers with the help of Zero. The Vaisesikas have no method to define zero first and then the numbers unity, duality etc. (or to define zero with the knowledge of unity, duality etc.) by means of relative understanding. It was Frege-Russel [2] definition of number which could enable one to define zero first and then other higher numbers.

Further we see that we can get only finite numbers, however large, in their system and as such the concept of infinity cannot be cognized. But Cantor (1845-1918) has shown how to deal with the infinite, and hence it is both desirable and possible to deal with the fundamental properties of numbers in some way which is applicable to finite as well as infinite numbers.

\section{Peano's definition of number}

Guiseppe Peano [3] believed like Pythagoras that the whole of mathematics could be deducted from numbers. Having reduced all traditional pure mathematics to the theory of natural numbers, the next step in the logical analysis was to reduce the theory itself to the minimum number of premises and undefined 
terms from which it could be derived. Peano showed that the entire theory of natural number could be derived from the three primitive ideas viz. 'zero', 'successor' and 'number' and the following five propositions.

(i) Zero is a number.

(ii) Successor of every number is also a number.

(iii) No two number have the same successor.

(iv) Zero is not a successor.

(v) If a property is possessed by zero and whenever it is possessed by any number. It is also possessed by its successor, then the property is possessed by all the numbers.

At present we shall simply discuss how he has defined number and how his definition has been improved by Russell [4]. Peano [3] gave the definition of number by abstraction, accepting that numbers are applicable essentially to classes (i.e collection) he defined number as a 'property of classes'. Two classes have the same number when one-one relation exists between their numbers. Two such classes are said to be 'similar' and the similarity relation has three properties viz reflexivity, symmetricity and transitivity. Peano told that when similarity relation holds between two terms, the two terms have a common property called their number [4].

But this definition is not satisfactory. For, all similar classes have the same number as a common property, so there is a many one relation which every class has to its number and to nothing else, In other words, we have a set of entities such that any class, and all other classes similar to it, have a certain many one relation to one and only one of the entities of the set. But there may be many such sets of entities and the definition of Peano by abstraction fails to define the number of class. The axioms of Peano do not enable us to know whether there exists any set of terms verifying the axioms. We want our number to be such that can be used for counting and this requires that our numbers should have a definite meaning, not merely that they have certain formal properties only. This definite meaning is defined only by the logical theory of arithmetic.

\section{Frege-Russell definition of number}

It was Frege [2] who first of all attempted to give a logical definition of numbers but none paid any attention to his views until Russell independently gave the same definition of number. in 1901. He did not define number by means of relative understanding (like the Vaisesikas) but he used two concepts viz. the similarity of sets' (which Peano also used) and the class of classes to define all numbers including zero Russell also introduced infinity through an axiom known as the 'Axiom of Infinity'. One might confuse the concept of number of a collection with its plurality. But this is not proper. Plurality is not a number but an instance of some particular number. For example, the trio of men, say, Ram, Shyam and Hari is an example of the number three, but the latter is not identical with the trio consisting of Ram, Shyam and Hari.

According to Frege and Russell, number is an attribute (unlike colour, taste etc. as it is according to the Vaisesikas)- it is defining property of a collection. There are two ways of defining a collection. One way is by 'extension'. i.e. enumeration and the other way is by 'intension', i.e. characterization. According to the extension method number of a collection is to be determined by counting the members of the collection. But this system suffers from two defects. The first defect is that counting is nothing else than labelling each member of a collection with a number and then it suffers from the defect of circularity. Secondly, numbers themselves form an infinite class and hence it is not always possible to enumerate or count the member of an infinite collection. Hence Frege and Russell attempted to define number by intension. Russell defines it in two steps, first he defines the concept of similarity of two sets and then he formulates the concept of number in terms of this similarity concept. He defines the number of a set to be "the class of all sets similar to it [5]. Similarity can be defined as in case of Peano, without 
the help of the concept of numbers. For example, in a country where polygamy is prohibited, one can say without counting that the set of husbands is similar to the set of wives, for every husband has a wife and every wife has a husband uniquely. We need not count the actual number of the husbands or the wives. The second part of this definition describes number as a 'class of classes' or more clearly, a 'class of similar classes' At the first sight it appears paradoxical to accept number as 'class of classes' and Peano never accepted this definition. But if we treat class as a concept and not as a collection, then a number is really defined as a common property of a set of similar classes and nothing else. This view removes the appearance of the paradox to a great extent. Further it may be seen that this definition allows the deduction of all the usual properties of numbers, finite as well as infinite, and is the only one which is possible in terms of the fundamental concept of general logic [4].

Frege-Russell definition too is far from being satisfactory. Firstly, we find that like Peano they too have based their definition on the concept of "similarity of sets.' But it is not always easy to establish similarity between two collections. If we have two collections, say a collection of cups and another collection of saucers, we can verify the similarity between these collections by placing each cup on a saucer and finding that no saucer is left without cup. Here one-one correspondence is established. But if the cups are closed in one box and saucers in another box, no such correspondence is visualized unless the boxes are opened and the cups are placed on saucers. The question arises whether similarity existed between the two collection before the cups were actually placed on the saucers obviously we feel that such similarity exists from before, so we see that it is possible to exhibit the correspondence between two sets only when similarity exists between them from before. Hence we conclude that correspondence simply verifies similarity. It is not at all possible to establish a correspondence between these collections. Hence the proposed definition of similarity gives only a sufficient but not necessary condition of similarity and restricts the meaning of similarity too narrowly. Secondly, in order to determine similarity between two classes, we have to know the proper meaning of the word 'class'. It has two meanings viz. 'list' and 'concept'. If it means list i.e. a collection, then correspondence can be established between the members of two collectors only if they have equal number of members. But if class means concept, then two concepts can be put in one-one correspondence, even though one set is an extension of the other, i.e., even if two classes are not equi-numbered.

According to Frege two sets must either be similar or not similar from a purely logical basis. But if someone is asked to tell the number of stars twinkling in the sky in dark night at particulars moment, he has no method to determine the number; for while establishing correspondence with any other collection, say, a collection of mustard seeds, some stars will disappear and some new ones will appear without his notice. At best he can say that there are many stars. Hence we find that sometimes we have to indicate the number of a collection by numerals 'many', 'very many' and 'few'. But Frege seems to pay no attention towards this aspect.

From the second part of Frege's definition and the manner of description for the number zero, one, two, etc. It appears that number states something about the concept and not about the counted things themselves. But do we always remember this fact in our language or statements? In any command, say, ' 3 books' the command does not say that the class of books to be asked for is 'an element of the class three'. Our command or language is unaware of this interpretation. It cannot be expressed in the subject predicate form. Frege-Russell definition unnecessarily restricts the concept of number to the subject predicate form of own preposition [6].

\section{Concluding remarks}

The Vaisesikas accepted number as a real quantity inhering in all substances. They realized that all higher numbers are formed by means of relative understanding of unit as a basic number, but they have not 
defined zero. Peano defined number as a property of classes, but this definition is not satisfactory, as number should have a definite logical meaning. Frege and Russell gave the logical definition of number. They have defined number as a common property of a set of similar classes and nothing else, but this definition too is far from being satisfactory, because it is not always easy to establish similarity between two collections. Thus we conclude that the number states something about the concept and not about the counted things themselves.

\section{References}

[1] B.B. Dutta, A.N. Singh, Hindu Ganit Shastra Ka Itihas, 1956.

[2] G. Frege, The basic laws of arithmetic, University of California Press, 1967.

[3] H. C. Kennedy, Peano: Life and Works of Guiseppe Peano, D. Radial Pub., 1980.

[4] B. Russell, The principle of Mathematics, George Allen and Unwin London, 1956.

[5] B. Russell, Introduction to Mathematical Philosophy, Macmillan, New York, 1924.

[4] F. Waismann, Introduction to Mathematical thinking, Harper \& Brother, New York, 1959. 